\title{
A comparative study of host response to three Mycobacterium tuberculosis PE_PGRS proteins
}

Correspondence

Michael J. Brennan

mbrennan@aeras.org

Received 24 April 2008

Revised 8 July 2008

Accepted 23 July 2008

\author{
Prachi P. Singh, Marcela Parra, Nathalie Cadieux and Michael J. Brennan† \\ Center for Biologics Evaluation and Research, Food and Drug Administration, Bethesda, MD \\ 20910, USA
}

\begin{abstract}
Three Mycobacterium tuberculosis proteins, PE_PGRS 16 (Rv0977), PE_PGRS 26 (Rv1441c) and PE_PGRS 33 (Rv1818c), were expressed in Mycobacterium smegmatis and used to investigate the host response to members of this unique protein family. Following infection of macrophages with the recombinant $M$. smegmatis $(M s)$ strains, Ms-PE_PGRS 33 and $M s^{-}$ PE_PGRS 26 were significantly more persistent ( 4.4 and 4.2 log c.f.u.) compared with MsPE_PGRS 16 (3.4 log c.f.u.) at day 6. Similarly, after infection of mice, Ms-PE_PGRS 33 and MsPE_PGRS 26 persisted at significantly higher levels in the spleen (3.5 and 3.2 log c.f.u.) and liver ( 3 and $2.6 \log$ c.f.u.) compared with Ms-PE_PGRS 16 in the spleen (2 log c.f.u.) and in the liver (1 log c.f.u.) at day 10. Increased persistence of Ms-PE_PGRS 33 and Ms-PE_PGRS 26 was associated with cell death and increased release of lactate dehydrogenase in macrophage cultures as well as increased levels of IL-10 and, in contrast, lower levels of IL-12 and NO both in vitro and in mouse splenocytes. Conversely, poor survival of Ms-PE_PGRS 16 was associated both in macrophage cultures and in vivo with higher levels of NO and IL-12. All three PE_PGRS proteins were found to be cell-surface antigens, but immunization of mice with these PE_PGRS antigens as DNA vaccines showed no protection in a TB aerosol challenge model. In general, the results suggest that variable expression of different PE_PGRS proteins within host cells can affect either the fate of the mycobacterial pathogen or that of the host during infection and point to the importance of studying the expression and function of individual members of the PE_PGRS gene family of M. tuberculosis.
\end{abstract}

\section{INTRODUCTION}

The deciphering of the Mycobacterium tuberculosis genome revealed the existence of a unique pe_pgrs gene family encoding proteins consisting of two major domains: a PE domain linked to a PGRS domain, which contains numerous glycine- and alanine-rich repeats (Cole et al., 1998; Brennan, 2002). There is much interest in this gene family since, to date, its members have been found only in the genomes of mycobacteria and are mostly restricted to pathogenic mycobacteria (Brennan et al., 2004). Understanding the function, localization, regulation and host response to PE_PGRS proteins is vital for determining what role these proteins may play in mycobacterial pathogenesis. To date, evidence indicates that certain

tPresent address: Aeras Global TB Vaccine Foundation, 1405 Research Boulevard, Suite 300, Rockville, MD 20850, USA.

Abbreviations: BCIP, 5-bromo-4-chloro-3-indolyl phosphate; BMMO, bone marrow macrophages; $\mathrm{LDH}$, lactate dehydrogenase; NBT, nitroblue tetrazolium; PE, proline-glutamine; PGRS, polymorphic GC-rich sequences; OADC, oleic acid-albumin-dextrose-catalase; TB, tuberculosis.

A table of primers and a supplementary figure are available with the online version of this paper.
PE_PGRS proteins can be specifically expressed in granulomas (Ramakrishnan et al., 2000), can be found at the surface of mycobacteria, where they may influence infectivity of host cells (Brennan et al., 2001; Espitia et al., 1999; Delogu et al., 2004), and can induce host cell necrosis (Dheenadhayalan et al., 2006a) or apoptosis (Basu et al., 2007; Balaji et al., 2007). Regulatory mechanisms affecting pe_pgrs gene expression are unknown, although various PE_PGRS proteins have been shown to be differentially expressed by $M$. tuberculosis both in vivo (Delogu et al., 2006) and in vitro (Dheenadhayalan et al., 2006b; Flores \& Espitia, 2003). Talarico et al. $(2005,2008)$ have reported that in-frame insertions/deletions and frameshifts can be frequently observed in a number of pe_pgrs genes in clinical isolates of $M$. tuberculosis, with most of the mutations occurring within the PGRS repeat domain. This supports the contention that pe_pgrs genes may be a source of antigenic variation for M. tuberculosis (Cole et al., 1998), but much remains to be revealed about the expression and function of individual members of the multigene PE_PGRS family.

In this study, we chose to compare three PE_PGRS members, PE_PGRS 16, PE_PGRS 26 and PE_PGRS 33, 
since there is some previous evidence that genetic variation can occur in these genes (Talarico et al., 2005, 2008) and that their expression may be regulated by different mechanisms (Dheenadhayalan et al., 2006b). PE_PGRS 26 was chosen for comparison since it represents a typical member of more than 60 PE_PGRS family members in both sequence and size while PE_PGRS 16 is representative of a small subset of PE_PGRS members which carry a unique domain linked at the $\mathrm{C}$ terminus to the PGRS domain. PE_PGRS 33 is one of the most extensively studied PE_PGRS proteins (Brennan et al., 2001; Delogu et al., 2004; Dheenadhayalan et al., 2006a; Basu et al., 2007; Balaji et al., 2007) and we used it to compare with the other two family members. Mycobacterium smegmatis strains expressing the PE_PGRS proteins were used to investigate the host response of macrophages and mice to infectivity with these strains and the effect of the PE_PGRS expression on the mycobacteria and the host cells. M. smegmatis is a non-pathogenic fastgrowing soil saprophyte that lacks PE_PGRS proteins. Hence it serves as a good model system to study the role of specific PE_PGRS proteins via construction of recombinant strains. Comparative studies on the three PE_PGRS antigens were also performed to determine if they may be useful targets for developing vaccines against tuberculosis (TB).

\section{METHODS}

Construction of plasmids and recombinant strains in $\boldsymbol{M}$. smegmatis. Full-length pe_pgrs 26 and pe_pgrs 33 were PCR amplified and cloned in the shuttle vector pMV261Nde-I under the hsp60 promoter between the NdeI and BamHI sites; full-length pe_pgrs 16 was cloned between the NdeI and HindIII sites in the same vector, using the primers listed in Supplementary Table S1, available with the online version of this paper. This plasmid is derived from pMV261 (the vector used in previous studies for pe-pgrs 33; Dheenadhayalan et al., 2006a); it has an NdeI site introduced to allow cloning of genes without fusing them to the $\mathrm{N}$-terminal portion of the Hsp60 protein and has a kanamycin-resistance gene replaced by a hygromycin-resistance gene from plasmid pYUB854. The recombinant plasmids were electroporated into $M$. smegmatis and colonies were selected on 7H11 agar plates (Difco) containing 10\% OADC (oleic acid-albumin-dextrose [glucose]-catalase enrichment) (BBL Middlebrook) and $50 \mu \mathrm{g}$ hygromycin B ml ${ }^{-1}$ (Sigma). The expression of pe_pgrs genes was confirmed by real-time RT-PCR and immunoblotting. For transfection and DNA vaccine studies each of the full-length pe_pgrs 26 and pe-pgrs 33 genes were cloned in the mammalian expression vector pJW4303 between the HindIII and BamHI sites, and pe_pgrs 16 was cloned between the HindIII and AvrII sites in the same vector. The sequence of the inserts was validated by automated DNA sequencing.

Quantitative real-time PCR. Total RNA was extracted from mycobacterial cultures or from mouse organ homogenates using a RNeasy minikit (Qiagen). Briefly, mouse organs were macerated in DMEM and centrifuged. The pellet was resuspended in Ack RBC lysis buffer followed by washes with DMEM. The pellet was finally resuspended in RLT buffer supplemented with $\beta$-mercaptoethanol and the lysate was passed through a 20 gauge needle fitted to an RNase-free syringe five times for homogenization. One volume of $70 \%$ ethanol was added to the lysate and a sample was loaded onto the RNeasy colum. This was followed by washes with buffers RW1 and RPE from the kit and the RNA was eluted with RNase-free water. After quantification, RNA samples were treated with DNase I (Invitrogen) and subjected to cDNA synthesis using the Superscript First-Strand Synthesis system for RT-PCR (Invitrogen), following the manufacturer's instructions. Quantitative expression of the pe_pgrs genes and 16S rRNA gene was performed by real-time PCR using Cepheid's real-time PCR smart cycler. PCR was performed in a $25 \mu \mathrm{l}$ reaction volume containing $12.5 \mu \mathrm{l} 2 \times$ Real-time Premix ExTaq from TaKaRa, each primer pair and Taqman probe at a concentration of $0.5 \mu \mathrm{M}$. Samples were subjected to an initial step of $50{ }^{\circ} \mathrm{C}$ for $2 \mathrm{~min}$ and subsequent denaturation at $95{ }^{\circ} \mathrm{C}$ for $5 \mathrm{~min}$. This was followed by 40 cycles each of $95{ }^{\circ} \mathrm{C}$ for $15 \mathrm{~s}$ and $60{ }^{\circ} \mathrm{C}$ for $1 \mathrm{~min}$. Each reaction was performed in triplicate and the mean threshold cycle $\left(C_{\mathrm{t}}\right)$ was determined for each sample and plotted against the cDNA concentration input to calculate the slope. Amplification efficiency was then calculated using the formula $E=10^{(-1 / \text { slope) }}$ (Pfaffl, 2001). All PCRs were performed with equal efficiencies; hence relative mRNA expression levels of pe_pgrs genes were directly normalized against $16 S \quad r R N A$ expression using the formula $2^{-\Delta C_{\mathrm{t}}}$, where $\Delta C_{\mathrm{t}}=\left(C_{\mathrm{t}}\right.$ target gene $)-\left(C_{\mathrm{t}}\right.$ reference gene $)$.

Transfections and immunoblotting. For transfection of eukaryotic cells with nucleic acid-based constructs, human rhabdomyosarcoma cells were seeded in 24-well plates at a density of $1.5 \times 10^{5}$ per well. The following day, lipofectamine 2000 (Invitrogen) was used to transfect cells with $1 \mu \mathrm{g}$ of the different pe_pgrs constructs in the mammalian expression vector pJW4303, following the manufacturer's recommendations and using the vector as a control. After $6 \mathrm{~h}$ the transfection mixture was removed and replaced with fresh growth medium. Protein expression was monitored after 24 h by $10 \%$ SDSPAGE and immunoblotting. PE_PGRS proteins were detected using a recently established anti-PE_PGRS mAb, 7C4.IF7, which will be described in more detail elsewhere (M. Parra and others, unpublished results) or mouse sera diluted in PBS-milk as the primary antibodies; alkaline phosphatase conjugated goat anti-mouse IgG was used as secondary antibody. The blots were developed using the colorimetric substrate NBT/BCIP (KPL). For immunofluorescence studies, the anti-PE_PGRS mAb 7C4.IF7 and goat anti-mouse antibody conjugated to Alexafluor 488 were used as primary and secondary antibody, respectively, and photographs at a final magnification of $\times 1000$ were taken with a Nikon Optiphot- 2 microscope equipped with a digital camera and using the FITC-GFP channel adjusted with phase-contrast to show the fluorescence superimposed on the background of mycobacteria.

Infection of murine bone marrow macrophages (BMMO) with recombinant $\boldsymbol{M}$. smegmatis. BMMO were isolated from femurs of healthy $\mathrm{C} 57 \mathrm{BL} / 6 \mathrm{~J}$ mice and cultured in complete DMEM as previously described (Delogu \& Brennan, 2001). Macrophages were infected with vector pMV261Nde-I and different recombinant $M$. smegmatis strains at a m.o.i. of 5:1 (bacteria:macrophage). A few wells containing coverslips were used for Giemsa staining to visualize cytoplasmic and nuclear details microscopically following infection. At various time points ( $4 \mathrm{~h}$ and days 3, 6 and 10), the culture supernatants were collected and stored at $-80{ }^{\circ} \mathrm{C}$ for in vitro tests. The BMMO were then washed once with PBS to remove extracellular bacteria, lysed in $0.1 \%$ saponin (Sigma) by incubating for $3 \mathrm{~min}$ at room temperature, serially diluted in PBS containing $0.05 \%$ Tween 80 and plated on Middlebrook 7H11 agar (Difco) plates supplemented with $10 \% \mathrm{OADC}$ and $50 \mu \mathrm{g}$ hygromycin $\mathrm{B} \mathrm{ml}^{-1}$. Colony forming units (c.f.u.) were determined as a measure of the intracellular survival of recombinant $M$. smegmatis. Statistical analyses were performed using a one-way analysis of variance, and significant differences (including determination of $P$ values) between the means were measured by Tukey's test using GraphPad Prism 4 software. 
In vitro assays. The extent of cytolysis of infected macrophages was determined by measuring the release of lactate dehydrogenase (LDH) in the supernatants of infected macrophages using the CytoTox 96 non-radioactive cytotoxicity assay kit (Promega) as described in the manufacturer's instructions. Samples were read at $490 \mathrm{~nm}$ on a VersaMax tunable microplate reader (Molecular Devices). Background release of LDH was calculated using supernatants of uninfected cells, and maximum release of LDH from the complete lysis of uninfected BMMO. The percentage of cell death was estimated by the following formula: [release of LDH from infected cells $\left(A_{490}\right)$ / maximum LDH release $\left.\left(A_{490}\right)\right] \times 100$.

The amount of IL-10 and IL-12 in the culture supernatants of infected BMMO was assayed using BD OptEIA mouse cytokines kits (BD Pharmingen) and the ELISA was performed in accordance with the manufacturer's instructions. Samples were read at $450 \mathrm{~nm}$ on a VersaMax tunable microplate reader with a reference wavelength of $650 \mathrm{~nm}$. Cytokines were quantified by comparison with specific recombinant standards using a log-log fit regression in the SoftMax Pro ELISA analysis software (Molecular Devices). To determine nitrite released by macrophages (as a measure of nitric oxide production), culture supernatants were tested with Griess reagent (Promega) and the absorbance was measured at $520 \mathrm{~nm}$. In all cases standard curves were plotted and samples were tested in triplicate in order to measure statistically significant differences.

In vivo infection in mice with recombinant $M$. smegmatis. Five C57BL/6 J female mice per group were each infected by intraperitoneal injection with $2 \times 10^{7}$ c.f.u. of $M$. smegmatis strains containing vector only, or expressing PE_PGRS 33, PE_PGRS 16 or PE_PGRS 26, and c.f.u. were determined as previously described (Dheenadhayalan et al., 2006a) on days 2, 4 and 10. For studying in vivo immune responses following infection with recombinant strains, spleens were removed at day 4 and homogenized in DMEM. The homogenate was treated with RBC lysing Ack buffer (Quality Biological) followed by three washes with incomplete DMEM. Splenocytes were cultured in a 24 -well microplate in complete DMEM for 3 days and the supernatants were tested for release of nitrite and cytokines.

Extraction of recombinant proteins in $M$. smegmatis with the detergent Genapol. To determine if PE_PGRS proteins are found at the surface of recombinant $M$. smegmatis strains a method described by Cascioferro et al. (2007) was used. Bacteria were cultured for 3 days, washed once with $1 \times$ PBS, and one half of the pellet (each half contained $10 \mathrm{mg}$ of bacteria) was incubated in $50 \mu \mathrm{l} \mathrm{PG05}$ buffer containing $0.5 \%(\mathrm{v} / \mathrm{v})$ Genapol for $30 \mathrm{~min}$ at $50{ }^{\circ} \mathrm{C}$. The other half of the sample was used as a control. Samples were centrifuged twice for $10 \mathrm{~min}$ at $4{ }^{\circ} \mathrm{C}$ at $6000 \mathrm{~g}$ and the supernatant was checked for PE_PGRS protein content by Western blots using the 7C4.IF7 $\mathrm{mAb}$ as primary and goat anti-mouse alkaline phosphatase as the secondary antibody and developed with the phosphatase substrate BCIP/NBT (KPL).

Immunizations and aerosol challenge. C57BL/6 J female mice (five per group) were immunized three times at 3 week intervals with $100 \mu \mathrm{g}$ each of the pe_pgrs 16, pe_pgrs 26 and pe_pgrs 33 DNA vaccines intramuscularly using vector pJW4303 as a control. For the BCG immunizations, the mice received $1 \times 10^{6}$ c.f.u. subcutaneously at the beginning of the experiments. Three weeks after the last immunization, mice were challenged for the protection study with approximately 200 c.f.u. of M. tuberculosis Erdman by aerosol. Four weeks later mice were sacrificed and bacterial colonization was determined in spleens and lungs as described previously (Parra et al., 2006). All animal protocols were approved by the Center for Biologics Evaluation and Research/Food and Drug Administration Institutional Animal Care and Use Committee.
RESULTS

\section{Expression of PE_PGRS proteins in M. smegmatis}

The three PE_PGRS proteins chosen for comparative studies are shown schematically in Fig. 1. PE_PGRS 26 and PE_PGRS 33 are representative of the more typical PE_PGRS proteins containing a PE domain linked to a glycine/alanine-rich PGRS domain, while PE_PGRS 16 is an example of an atypical subset of the PE_PGRS family of genes which contains a unique sequence at the $\mathrm{C}$ terminus following the PGRS domain. PE_PGRS 33 and PE_PGRS 26 are very similar in both size (491 and $498 \mathrm{aa}$ ) and amino acid sequence (53\% identical), while PE_PGRS 16 is a larger protein (923 aa) that has a unique C-terminal domain of 270 aa linked to the PGRS domain. To construct recombinant $M$. smegmais strains, full-length M. tuberculosis pe-pgrs 16, pe-pgrs 26 and pe-pgrs 33 were cloned in shuttle vector pMV261Nde-I under the control of the $h s p 60$ promoter, which results in constitutive expression of the gene of interest, and the resulting constructs were electroporated into M. smegmatis. The expression of these PE_PGRS proteins by recombinant strains was confirmed by real-time RT-PCR and immunoblotting (Supplementary Fig. S1) and the growth rates of these strains in axenic culture were also found to be similar (data not shown).

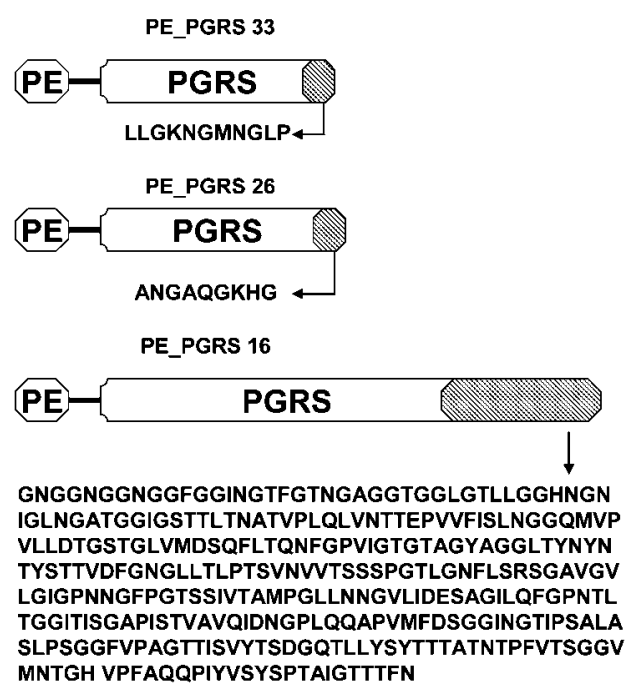

Fig. 1. A schematic representation of the three PE_PGRS proteins used for comparative studies. PE_PGRS 33 and PE_PGRS 26, which are $53 \%$ identical in both the PE and PGRS domains, are similar in size (491 and 498 aa) and contain a small unique C-terminal sequence of $9-11$ aa. PE_PGRS 16 is a larger protein (923 aa) with a similar PE and PGRS domain but it contains an extended unique $\mathrm{C}$-terminal region of 270 aa. 


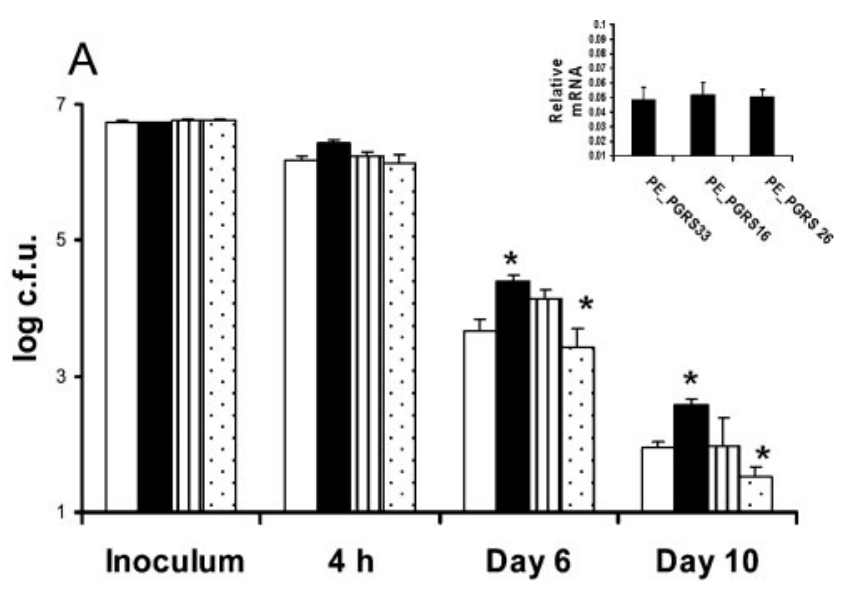

B

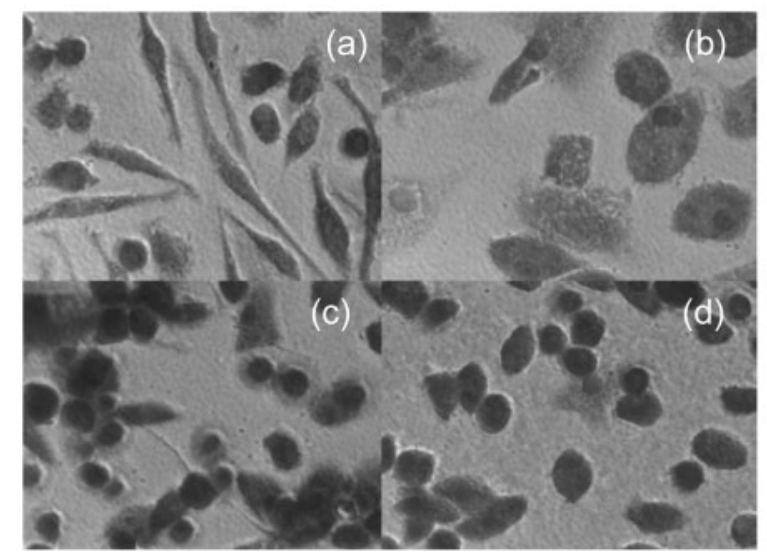

\section{Persistence and cellular immune responses following infection of macrophages with recombinant $\boldsymbol{M}$. smegmatis expressing PE_PGRS proteins}

We have previously reported that recombinant $M$. smegmatis expressing PE_PGRS 33 shows increased persistence in mouse tissues and murine macrophages (Dheenadhayalan et al., 2006a). In the present study we compared the ability of recombinant $M$. smegmatis strains expressing three different PE_PGRS proteins to survive in macrophages. Mouse primary bone marrow macrophages (BMMO) were infected with the recombinant M. smegmatis strains expressing different PE_PGRS proteins at a m.o.i. of 5:1 (bacteria: macrophage). Determinations of c.f.u. of the inoculum used for infection demonstrated that a similar number of bacteria $\left(5.5 \times 10^{6}\right.$ c.f.u. $)$ per well infected the BMMO. Importantly, the relative mRNA expression levels for each PE_PGRS protein expressed by the recombinant strains in infected BMMO were similar as demonstrated by real-time RT-PCR (Fig. 2A, inset). Following infection, Ms-PE_PGRS 33 showed the highest persistence ( $0.8 \log$ c.f.u. higher than the control strain) over a period of 6-10 days (Fig. 2A), while Ms-
Fig. 2. Infection of macrophages with recombinant $M$. smegmatis strains. Inset: real-time RT-PCR was used to measure mRNA expression levels in BMMO infected with $M$. smegmatis expressing PE_PGRS 33, PE_PGRS 16 and PE_PGRS 26 for 10 days. mRNA levels for three samples each were determined as described in Methods and are expressed relative to 16S rRNA. (A) Primary bone marrow macrophages from C57BL/6 mice were infected at a m.o.i. of $5: 1$ with recombinant $M$. smegmatis expressing the vector only (white bars), PE_PGRS 33 (black bars), PE_PGRS 26 (striped bars) and PE_PGRS 16 (dotted bars). The initial inoculum for each strain was determined and cells from four wells per experimental group were collected for c.f.u. determinations at $4 \mathrm{~h}, 6$ days and 10 days after infection. The error bars indicate SEM and the asterisks denote significant differences between Ms-PE_PGRS 33 and Ms-PE_PGRS 16 infected cultures $(P<0.001)$. Similar results were obtained in four independent experiments. $(B)$ In microscopic observations $(\times 1000)$ of infected macrophages at day 6, Ms-PE_PGRS 33-infected cells are necrotic and associated with large numbers of bacteria (b) compared with cells infected with $M$. smegmatis expressing the vector only (a) or Ms-PE_PGRS 16 (c), which are mostly healthy, Some necrotic cells and bacteria are visible in macrophage cultures infected with Ms-PE_PGRS 26 (d).

PE_PGRS 26 survived at $0.6 \log$ c.f.u. higher than vector alone at day 6 but showed persistence similar to the vector at day 10. Interestingly, Ms-PE_PGRS 16 showed significantly lower persistence than both PE_PGRS 33 (1 log c.f.u. less) and the strain harbouring the vector $(0.5 \log$ c.f.u. less) at day 10. Microscopic observations at day 6 revealed interesting differences in macrophage cultures infected with M. smegmatis expressing different PE_PGRS proteins (Fig. 2B). The BMMO infected with $M$. smegmatis harbouring vector alone appeared healthy while a large number of the macrophages infected with Ms-PE_PGRS 33 were necrotic, associated with a distorted nucleus and ruptured cells leading to the release of cytosolic content and bacteria into the extracellular environment. Macrophages infected with Ms-PE_PGRS 26 showed lower levels of necrosis compared with $M s$-PE_PGRS 33-infected cells, and macrophages infected with Ms-PE_PGRS 16 were mostly healthy, with fewer visible bacteria.

The macrophage infectivity data suggested that the host cells may be responding differently to $M$. smegmatis expressing the different PE_PGRS proteins. To quantify levels of cytolysis in macrophage cultures, the amount of LDH was measured in the culture supernatants over time. As evident in Fig. 3(A), there was a significantly higher release of $\mathrm{LDH}(\sim 45 \%)$ by day 6 in macrophages infected with Ms-PE_PGRS 33, as previously reported (Dheenadhayalan et al., 2006a). In macrophages infected with $M s$-PE_PGRS 26, levels of LDH were relatively high, $30-35 \%$ following 6 days of infection, but less than that induced by the PE_PGRS 33 protein. In contrast, macrophages infected with Ms-PE_PGRS 16 showed much lower levels of $\mathrm{LDH}$, ranging no higher than $10-15 \%$ over 
A
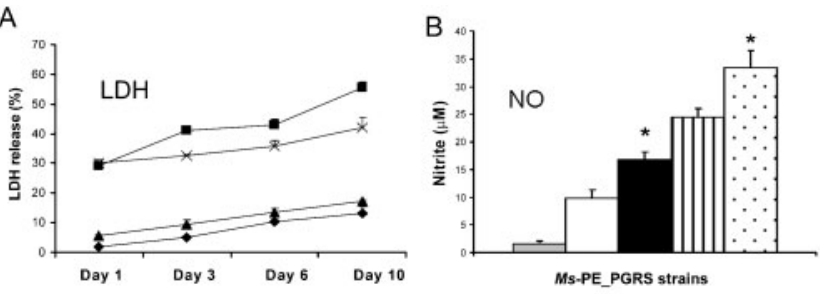

C

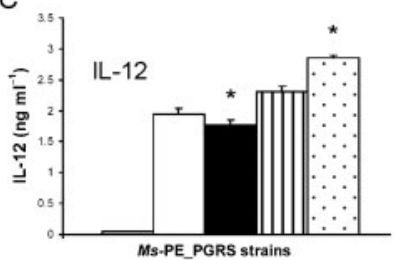

D

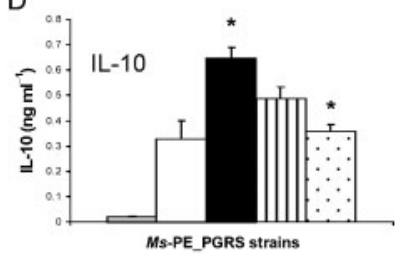

Fig. 3. Measures of cytolysis, intracellular killing and cytokine release following infection of macrophages with $M$. smegmatis strains expressing PE_PGRS proteins. Culture supernatants were collected from uninfected BMMO (grey bars) and from BMMO infected at a m.o.i. of $5: 1$ with $M$. smegmatis expressing the vector only (white bars, $>$ ) PE_PGRS 33 (black bars, $\mathbf{\square})$, PE_PGRS 26 (striped bars, $\times$ ) and PE_PGRS 16 (dotted bars, $\mathbf{\Delta}$ ), and the release of $\mathrm{LDH}$ at different time points $(\mathrm{A})$ and $\mathrm{NO}(\mathrm{B})$ cytokine IL12 (C) and IL-10 (D) were determined at day 3. Asterisks in B-D denote significant differences between Ms-PE_PGRS 33- and $M s$-PE_PGRS 16-infected cultures $(P<0.001)$. Similar results were obtained in three independent experiments.

10 days in culture, similar to the response to $M$. smegmatis harbouring the vector alone $(8-10 \%)$.

Since both reactive nitrogen intermediates and cytokines can control the intracellular fate of mycobacteria in host cells, we measured the levels of nitric oxide $(\mathrm{NO})$ and the cytokines IL-10 and IL-12 following infection of BMMO with the recombinant $M$. smegmatis strains expressing the PE_PGRS proteins. The highest NO production $(33.3 \mu \mathrm{M})$ was observed in macrophages infected with $\mathrm{Ms}$ PE_PGRS 16, which was followed by macrophages infected with Ms-PE_PGRS $26(25 \mu \mathrm{M})$ and then by $M s$ PE_PGRS $33(16.7 \mu \mathrm{M})$ after 3 days in culture (Fig. 3B). Macrophages infected with $M s$-PE_PGRS 16 released the highest amount of IL-12 $\left(2.8 \mathrm{ng} \mathrm{ml}^{-1}\right)$ and the lowest amount of IL-10 (0.32 $\left.\mathrm{ng} \mathrm{ml}^{-1}\right)$ at day 3 (Fig. 3C, D). Conversely, macrophages infected with Ms-PE_PGRS 33 released the highest levels of IL-10 $\left(0.68 \mathrm{ng} \mathrm{ml}^{-1}\right)$ and the least IL-12 $\left(1.4 \mathrm{ng} \mathrm{ml}^{-1}\right)$. Infection with Ms-PE_PGRS 26 induced $0.48 \mathrm{ng} \mathrm{IL}-10 \mathrm{ml}^{-1}$ and $2.1 \mathrm{ng} \mathrm{IL}-12 \mathrm{ml}^{-1}$. The data demonstrate an association between increased levels of $\mathrm{NO}$ and IL-12 and decreased survival for the $\mathrm{Ms}$ PE_PGRS 16 strain in macrophages. Conversely, lower levels of NO and IL-12 and increased levels of IL-10 are associated with better survival of the Ms-PEPGRS 33 and $M s$-PE_PGRS 26 strains.

\section{In vivo survival and cellular responses to recombinant $M$. smegmatis strains expressing different $M$. tuberculosis PE_PGRS proteins following infection of mice}

In order to study the fate of recombinant $M$. smegmatis expressing different PE_PGRS proteins following in vivo infection, we compared the survival of recombinant $M$. smegmatis expressing each of the three PE_PGRS proteins in comparison to the vector control in mouse tissues. C57BL/6 mice were infected intraperitoneally with $2 \times 10^{7}$ c.f.u. of each of the recombinant $M$. smegmatis strains. The relative mRNA expression levels for each PE_PGRS protein expressed by the recombinant strains in mouse organs at day 10 were similar as determined by real-time RT-PCR (Fig. 4A, inset). As shown in Fig. 4(A), there was a fairly rapid clearance of bacteria in the mouse lungs; however, $M s$-PE_PGRS 33 at $4.2 \log$ c.f.u. and $M s-$ PE_PGRS 26 at $3.6 \log$ c.f.u. persisted at higher levels in the lungs at day 4 compared with Ms-PE_PGRS 16 (3 log c.f.u.) and the vector control (2.7 $\log$ c.f.u.). Also, significantly higher numbers of $M s$-PE_PGRS 33 were found in spleen ( $3.5 \log$ c.f.u.) and in liver ( $3 \log$ c.f.u.) in comparison to the vector (2.4 and $2.1 \log$ c.f.u.) at day 10 (Fig. 4B, C). Persistence of Ms-PE_PGRS 26 was relatively lower than that of $M s$-PE_PGRS 33 but significantly greater than that of the vector alone, since $\mathrm{Ms}^{-}$ PE_PGRS 26 persisted at $0.5 \log$ c.f.u. higher than the vector in liver and $0.7 \log$ c.f.u. higher in spleen after 10 days of infection. However, Ms-PE_PGRS 16 showed the least persistence: approximately 1.5-2 log c.f.u. less than Ms-PE_PGRS 33 in all three organs and 0.4 and $1 \log$ c.f.u. less in the spleen and liver compared to the vector alone.

Interestingly, as observed in the in vitro studies using $\mathrm{BMMO}$, the splenocytes from mice infected with $\mathrm{Ms}$ PE_PGRS 16 released the highest levels of NO $(27.8 \mu \mathrm{M})$ and IL-12 $\left(0.4 \mathrm{ng} \mathrm{ml} \mathrm{m}^{-1}\right)$ in comparison to $\mathrm{Ms}$ PE_PGRS $26\left(13.9 \mu \mathrm{M}\right.$ and $\left.0.2 \mathrm{ng} \mathrm{ml} \mathrm{m}^{-1}\right)$ and $\mathrm{Ms}^{-}$ PE_PGRS $33\left(10.5 \mu \mathrm{M}\right.$ and $\left.0.16 \mathrm{ng} \mathrm{m}^{-1}\right)$ (Fig. 5). Conversely, splenocytes from mice infected with $\mathrm{Ms}$ PE_PGRS 33 resulted in the highest levels of IL-10 $\left(0.8 \mathrm{ng} \mathrm{ml}^{-1}\right)$ as compared to $M s$-PE_PGRS $26(0.5 \mathrm{ng}$ $\left.\mathrm{ml}^{-1}\right)$ and $M s$-PE_PGRS $16 \quad\left(0.27 \mathrm{ng} \mathrm{ml}^{-1}\right)$. These results demonstrate that the cellular responses to the three PE_PGRS proteins are similar following both in vitro and in vivo infection, with PE_PGRS 16 inducing the highest levels of NO and IL-12 whereas PE_PGRS 33 induces the highest levels of IL-10. Together with the persistence data these observations suggest that expression of PE_PGRS 33 by M. smegmatis in particular is associated with less induction of host factors associated with the killing of mycobacteria and better persistence, while expression of PE_PGRS 16 leads to poor survival which is associated with higher levels of NO and IL-12. 

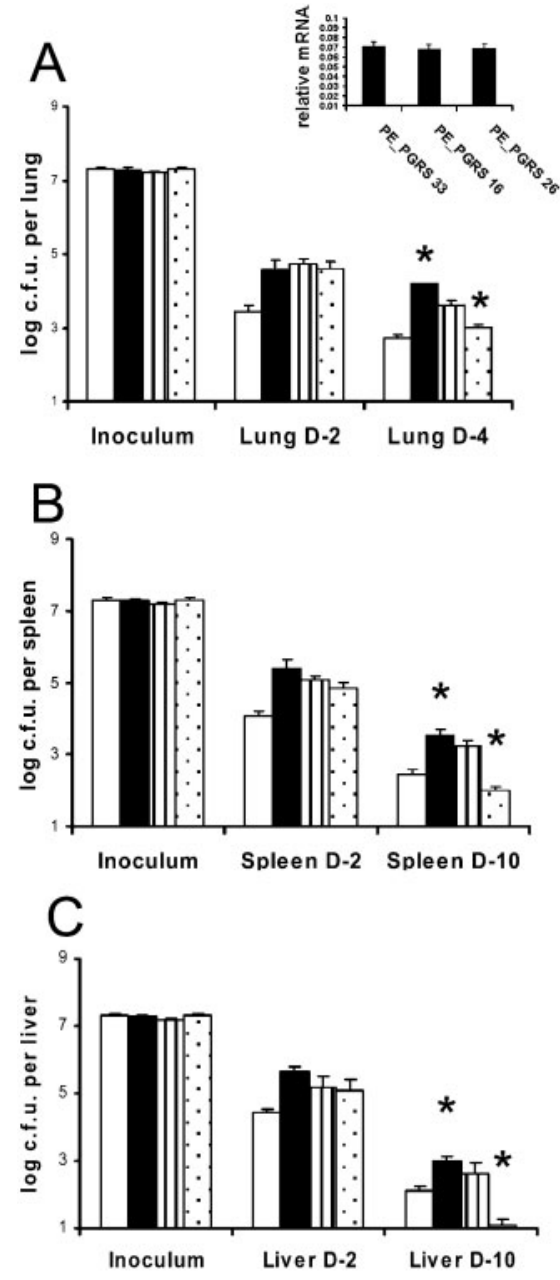

Fig. 4. Persistence of recombinant $M$. smegmatis strains expressing M. tuberculosis PE_PGRS proteins in mouse tissues. C57BL/ 6 mice were infected intraperitoneally with recombinant $M$. smegmatis expressing the vector only (white bars), PE_PGRS 33 (black bars), PE_PGRS 26 (striped bars) and PE_PGRS 16 (dotted bars). The initial inoculum for each strain was determined to be similar and mice were sacrificed after 2 and 4 days for c.f.u. determinations in the lung (A) and after 2 and 10 days for determination of c.f.u. in spleens (B) and liver (C). Asterisks denote a significant difference in log c.f.u. between $M s^{-}$ PE_PGRS 33- and Ms-PE_PGRS 16-infected mice $(P<0.001)$. Similar results were obtained in three independent experiments. Inset: Real-time RT-PCR was used to measure mRNA expression levels in mouse spleens infected with $M$. smegmatis expressing PE_PGRS 33, PE_PGRS 16 and PE_PGRS 26 for 10 days. mRNA levels for three samples each were determined as described in Methods and are expressed relative to 16S rRNA.

\section{Evidence that the three PE_PGRS proteins are cell surface antigens but are poor vaccine targets}

Previous evidence has indicated that certain PE_PGRS proteins can be found at the surface of mycobacteria, where they can interact with host cells and the immune system
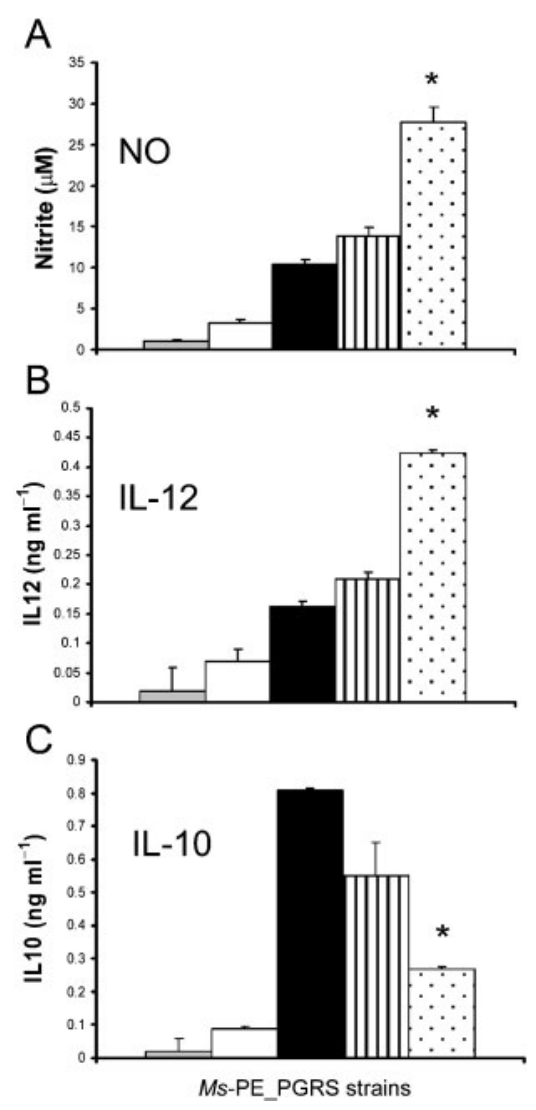

Fig. 5. Cellular responses in splenocytes cultured from mice infected with recombinant $M$. smegmatis strains expressing PE_PGRS proteins. Culture supernatants collected from splenocytes from uninfected mice (grey bars), mice infected with vector pMV261Nde-I (white bars), Ms-PE_PGRS 33 (black bars), MsPE_PGRS 26 (striped bars) and Ms-PE_PGRS 16 (dotted bars) were used to measure $\mathrm{NO}(\mathrm{A}), \mathrm{IL}-12$ (B) and $\mathrm{IL}-10$ (C) as described in Methods. The asterisks denote a significant difference between Ms-PE_PGRS 16 when compared with $\mathrm{Ms}^{-}$ PE_PGRS 33- or Ms-PE_PGRS 26-infected mice $(P<0.001)$. Similar results were obtained in three independent experiments.

(Brennan et al., 2001; Banu et al., 2002). In order to ascertain if the three PE_PGRS proteins expressed by the M. smegmatis strains are localized in the outer membrane, the recombinant $M$. smegmatis strains expressing the PE_PGRS proteins were treated with $0.5 \%$ Genapol at $50{ }^{\circ} \mathrm{C}$, a method that has been shown to extract loosely associated cell surface proteins from the mycobacterial cell wall (Heinz \& Niederweis, 2000; Cascioferro et al., 2007). Treatment with this mild detergent resulted in extraction of all three PE_PGRS proteins, which indicates that they are all loosely associated with the bacterial cell surface (Fig. 6A). In each case a control sample was used that consisted of untreated bacteria at $50{ }^{\circ} \mathrm{C}$ to demonstrate that extraction of proteins in the supernatant was not a result of treatment at high temperature (data not shown). An immunofluorescence study on recombinant M. smeg- 
matis strains using the anti-PE_PGRS mAb 7C4.IF7 as primary antibody and a goat anti-mouse antibody conjugated to Alexafluor 488 as secondary antibody in the reactions was performed. The $\mathrm{mAb}$ recognized clusters of extracellular antigen associated with the bacteria for all three PE_PGRS antigens (Fig. 7). A similar pattern was observed previously using anti-PE_PGRS 33 polyclonal antiserum, which recognized punctuate extracellular clusters of antigen associated with BCG (Brennan et al., 2001). These results provide additional evidence that PE_PGRS proteins can be found associated with the cell surface of mycobacteria.

Since the PE_PGRS 33, PE_PGRS 16 and PE_PGRS 26 proteins are expressed on the surface of mycobacteria, and may be targets for TB vaccines, nucleic acid-based pe_pgrs constructs were developed and used to immunize mice for M. tuberculosis challenge studies. Previously we have shown that a PE_PGRS 33 DNA vaccine does not protect against aerosol challenge with M. tuberculosis (Delogu \& Brennan, 2001). Since particularly PE_PGRS 16 can elicit different cytokine responses (see Figs 3 and 5) we were interested in determining the potential for DNA vaccines constructed from PE_PGRS 16 as well as PE_PGRS 26 to elicit an effective immunity in the mouse TB aerosol challenge model. Mice were immunized three times with each of the pe_pgrs DNA vaccines together with the vector control, challenged with $M$. tuberculosis Erdman strain (200 c.f.u.), and c.f.u. were determined in the lung and spleen 30 days after aerosol challenge. Immunization with BCG vaccine was used as a control and it reduced colonization of the lung by $0.87 \log$ c.f.u. and of the spleen by $0.94 \log$ c.f.u. 30 days after challenge (Table 1). None of the pe_pgrs vaccines gave protection as determined by colonization of the mouse tissues, and in fact the bacterial load in lungs 30 days after challenge was somewhat greater in mice immunized with pe_pgrs 33 (5.85 log c.f.u.) and pe_pgrs 26 (5.68 $\log$ c.f.u.) compared with the vector control (5.46 log

Table 1. Colonization of mouse lungs and spleen following immunization with PE_PGRS DNA vaccines and challenge with M. tuberculosis Erdman strain in the mouse TB aerosol model

C.f.u. were determined 30 days after challenge. The numbers in parentheses indicate the $\log$ c.f.u. differences compared with the vector control; an asterisk $\left(^{*}\right)$ indicates statistical significance $(P<0.001)$.

\begin{tabular}{|lll|}
\hline Vaccine group & \multicolumn{2}{c|}{ Colonization $(\log$ c.f.u. per organ) \pm SEM } \\
\cline { 2 - 3 } & \multicolumn{1}{c|}{ Lungs } & \multicolumn{1}{c|}{ Spleen } \\
\hline Naive & $5.57 \pm 0.18$ & $4.82 \pm 0.15$ \\
Vector (pJW4303) & $5.46 \pm 0.27$ & $4.79 \pm 0.08$ \\
BCG & $4.59 \pm 0.09(-0.87)^{\star}$ & $3.85 \pm 0.09(-0.94)^{*}$ \\
PE_PGRS 33 & $5.85 \pm 0.05(+0.39)$ & $4.79 \pm 0.15$ \\
PE_PGRS 26 & $5.68 \pm 0.19(+0.22)$ & $4.81 \pm 0.12$ \\
PE_PGRS 16 & $5.23 \pm 0.11(-0.23)$ & $4.78 \pm 0.18$ \\
\hline
\end{tabular}

c.f.u.) and pe_pgrs 16 (5.23 log c.f.u.) as shown in Table 1. Interestingly the sera from mice immunized with each pe_pgrs DNA vaccine not only showed reactivity with the respective recombinant protein but also cross-reacted with the other two PE_PGRS recombinant proteins (Fig. 6B). Also, the sera were reactive with recombinant PGRS protein but not with recombinant PE protein by immunoblotting (data not shown), which suggests that each PE_PGRS vaccine elicits antibodies directed against the common PGRS Gly/Ala-rich repeat domain (Banu et al., 2002; Delogu \& Brennan, 2001).

\section{DISCUSSION}

In this study, the non-pathogenic M. smegmatis was used as a vector to study how host cells respond to PE_PGRS proteins, since its genome does not contain the pe_pgrs family of genes, which are unique to pathogenic mycobacteria such as $M$. tuberculosis. Infection of mice and macrophages demonstrated that two of the three pe_pgrs genes studied, pe_pgrs 33 and pe_pgrs 26, gave similar results in that $M$. smegmatis strains expressing these proteins persisted better than the vector control, although $M$ s-PE_PGRS 33 survived better throughout both in vivo and in vitro. In contrast, Ms-PE_PGRS 16 showed significantly lower survival compared to the other $M s_{\text {- }}$ PE_PGRS strains and in some cases persisted less well than the M. smegmatis strain carrying the vector only. This was not due to differences in expression of the proteins since real-time RT-PCR showed that all three pe_pgrs genes are expressed equally in mouse tissues or macrophages. Aligned with this difference were the findings that, compared with Ms-PE_PGRS 33 and Ms-PE_PGRS 26, macrophages infected with $M s$-PE_PGRS 16 were also associated with increased levels of the nitrogen intermediate $\mathrm{NO}$, which is one mechanism used by macrophages for destroying intracellular mycobacteria, and also with lower levels of IL-10 and increased levels of IL-12, a cytokine profile that is typically more beneficial to the macrophage. Conversely, expression of PE_PGRS 33 and PE_PGRS 26 in $M$. smegmatis strains was associated with macrophage cell death as demonstrated by microscopic observations, increased LDH levels, relatively low NO levels, decreased levels of IL-12 and increased levels of IL-10 in the cultures. Since M. tuberculosis is known to promote host cell necrosis, which can lead to dissemination of the pathogen (Chen et al., 2006), these results suggest that the expression of certain PE_PGRS proteins such as PE_PGRS 26 and PE_PGRS 33 may play a role in this aspect of virulence.

When comparing previous results obtained using recombinant $M$. smegmatis strains in in vivo infection experiments with results described in Fig. 4, we observed that colonization of mouse lung tissues by the recombinant $M$. smegmatis strain expressing PE_PGRS 33 and the vector control was significantly increased compared to results published earlier (Dheenadhayalan et al., 2006a). This may be due to the fact that the pe_pgrs genes were cloned in a 


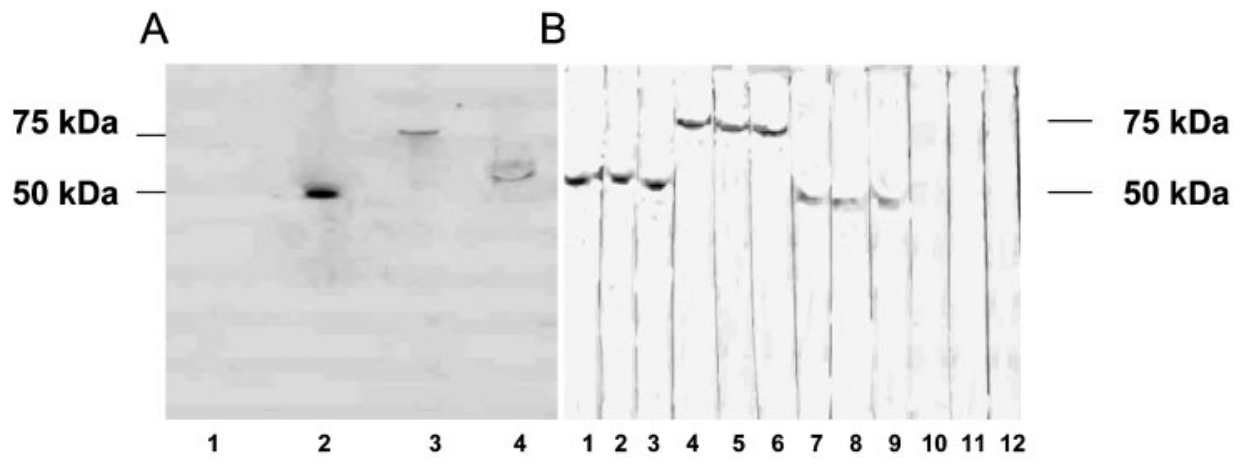

Fig. 6. PE_PGRS 16, PE_PGRS 26 and PE_PGRS 33 are found on the surface of recombinant $M$. smegmatis strains expressing these proteins, and elicit cross-reactive antibodies when DNA vaccines are used to immunize mice. (A) Western blot showing M. smegmatis harbouring vector pMV261Nde-I alone (lane 1), or expressing PE_PGRS 33 (lane 2), PE_PGRS 16 (lane 3) or PE_PGRS 26 (lane 4), extracted with $0.5 \%$ Genapol as described in Methods and probed with the anti-PE_PGRS mAb 7C4.IF7, which recognizes the PGRS domain of PE_PGRS antigens. (B) Western blots containing extracts of human rhabdomyosarcoma cells transfected with the pJW4303 vector expressing PE_PGRS 26 (lanes 1, 2 and 3), PE_PGRS 16 (lanes 4, 5 and 6), and PE_PGRS 33 (lanes 7, 8 and 9) as well as the vector only (lanes 10,11 and 12) were incubated with pooled sera from five mice $(1: 400$ dilution) immunized with the DNA vaccine constructs expressing PE_PGRS 33 (lanes $1,4,7$ and 10), PE_PGRS 16 (lanes 2, 5, 8 and 11) and PE_PGRS 26 (lanes 3, 6, 9 and 12). The proteins reactive with the antisera were detected with goat anti-mouse alkaline phosphatase as a secondary antibody and developed using the colorimetric substrate NBT/BCIP as described in Methods. The range of molecular mass as determined by protein standards is shown.

vector slightly different from that used in the previous study. In general, we have also found that survival of $M$. smegmatis in lung tissues lasts only 2-4 days, and is more variable than in spleens and liver. For this reason, experiments in mice needed to be repeated a number of times. However, it is important to note that the recombinant $M$. smegmatis infection data in spleen and liver tissues are reproducible and consistent with the previously published data.

As shown in Fig. 1, PE_PGRS 16 differs from the 'typical' PE_PGRS protein in that it contains a large C-terminal segment following the PGRS region that contains the characteristic Gly/Ala-rich repeat elements. This could account for the differences observed in our studies in comparison with PE_PGRS 33 and PE_PGRS 26, which share high similarity and contain the more typical PE and PGRS regions connected by a conserved linker region (Brennan \& Delogu, 2002). It is possible that the Cterminal segment found in PE_PGRS 16 directly induces the biological responses observed in our studies, or that it may cause conformational changes that alter the way the protein interacts with host cell factors. In genomic searches using the C-terminal segment of PE_PGRS 16 we have, to date, found no homologous domains of interest that would suggest a function for this unique doman, although interestingly C-terminal domains with approximately 38 $40 \%$ homology with PE_PGRS 16 also exist in PE_PGRS 35 and in PE 26 members of the PE family. Further studies are needed to determine the importance of these C-terminal domains for altering responses to
PE_PGRS proteins. While characterizing the DNA vaccines used in these studies, we also observed following transfection of macrophages that PE_PGRS 16 is localized differently from PE_PGRS33 and PE_PGRS 26: it occurs as discrete deposits dispersed throughout the cell, whereas PE_PGRS 33 and PE_PGRS 26 show a perinuclear distribution (P. P. Singh and others, unpublished data). This suggests that PE_PGRS 16 may interact with intracellular compartment components differently from PE_PGRS 33 and PE_PGRS 26 and that the antigen could be presented differently to the host immune system.

Expression and microarray studies have demonstrated that a number of pe_pgrs genes are regulated independently under various growth conditions (Voskuil et al., 2004). In previous studies, we have shown that PE_PGRS 16 and PE_PGRS 26 are inversely regulated, with expression of PE_PGRS 16 increasing after infection of mice or macrophages while the expression of PE_PGRS 26 decreases (Dheenadhayalan et al., 2006b). In contrast, the expression of PE_PGRS 33 remains fairly constant over time. Interestingly, pe_pgrs 16 belongs to an in vivo-expressed genomic island (Talaat et al., 2004) that also contains two other pe_pgrs genes, pe_pgrs 17 and pe_pgrs 18, and a mycobacterial persistence regulator mprA which is a twocomponent response transcriptional regulatory protein. It would be of interest to determine if the mprA system regulates the expression of pe_pgrs 16 and other pe_pgrs genes. Expression of PE_PGRS 16 has also been shown to be significantly upregulated following human brain microvascular endothelial cell invasion by M.tuberculosis 


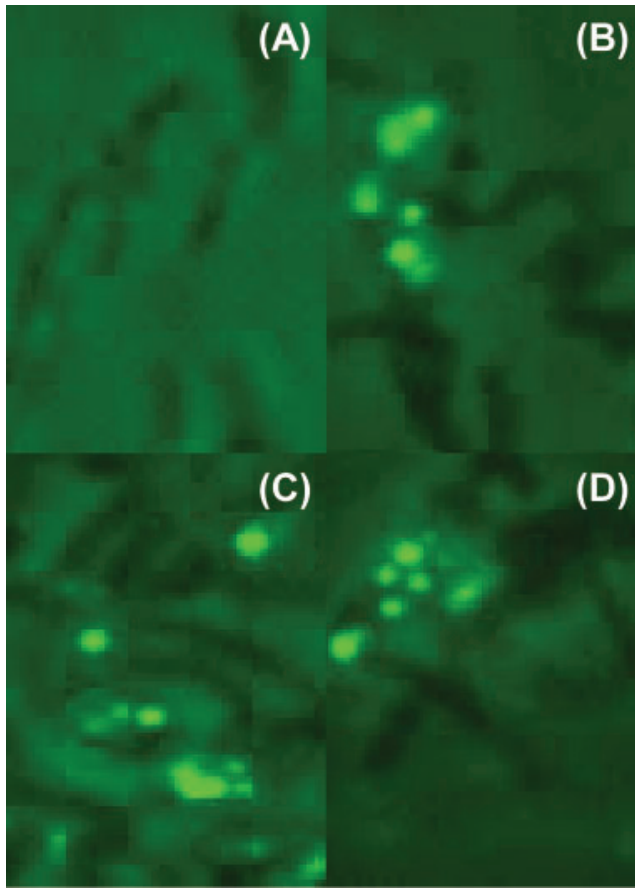

Fig. 7. Immunofluoresence using a $m A b$ to detect PE_PGRS antigen in cultures of recombinant $M$. smegmatis provides evidence that the PE_PGRS proteins are extracellular. Exponential-phase cultures of recombinant $M$. smegmatis expressing vector pMV261 Nde-1 (A), PE_PGRS 33 (B), PE_PGRS 16 (C) and PE_PGRS 26 (D) were incubated with the anti-PE_PGRS mAb 7C4.IF7 as primary and Alexafluor 488 as the secondary antibody. Extracellular localization of PE_PGRS antigen is observed in (B-D). Magnification $\times 1000$.

strain H37 Rv used as a model of CNS tuberculosis (Jain et al., 2006). In contrast to our previous findings (Dheenadhayalan et al., 2006b), Williams et al. (2007) have shown that expression of M. tuberculosis PE_PGRS 26 is significantly upregulated following induction of sigma factor F, which may help mycobacteria survive under stressful environmental conditions. The differences in host cell responses observed in the present studies using PE_PGRS 16 compared to PE_PGRS 33 and PE_PGRS 26 suggest that regulated expression of different PE_PGRS proteins by $M$. tuberculosis could affect the fate of the mycobacterial pathogen as well as the response by the host to infection. However, it remains to be determined how increased expression of PE_PGRS 16 in host cells could favour the mycobacteria and, conversely, how decreased expression of PE_PGRS 26 may benefit the bacterium. In fact, the initial data reported here suggest that this expression scenario would favour the host; this is a paradox that remains to be investigated. The ability of PE_PGRS 33 to induce cytolysis and enhance persistence of mycobacteria both in vitro and in vivo has been confirmed in these studies and suggests, as did earlier work using a transposon mutant in PE_PGRS 33 (Brennan et al., 2001), that expression of this PE_PGRS protein may be critical for maintaining the infectivity of the mycobacterium.

Talarico et al. $(2005,2008)$ have included pe_pgrs 16, pe_pgrs 26 and pe_pgrs 33 in their genetic studies and have shown that significant insertions and deletions occur mostly in the PGRS region of all three pe_pgrs genes in clinical isolates of $M$. tuberculosis. Most mutations in pe_pgrs 33 did not alter the reading frame whereas numerous frameshifts were observed in the case of pe_pgrs 16 and pe_pgrs 26. These results indicate that alterations in PE_PGRS proteins can occur through mechanisms of genetic recombination as well as via regulation of gene expression that could lead to changes in function and host recognition. These variations could reflect the differences in the ability of the pathogen to persist in the host.

Earlier work by our laboratory and others has shown that a number of PE_PGRS proteins, including PE_PGRS 33 (Delogu et al., 2004) and PE_PGRS 26 (Banu et al., 2002), can be found at the bacterial surface. This may be important for a possible role for PE_PGRS proteins in interacting with host cells to promote infectivity, for interacting with the host immune system or as important structural components of the cell wall (Brennan et al., 2004). Here we have shown that PE_PGRS 16, PE_PGRS 26 and PE_PGRS 33 can be found at the cell surface when expressed in $M$. smegmatis. The presence of PE_PGRS antigens at the surface of mycobacteria also suggests that they could be targets for new vaccines and drugs. Our previous study (Delogu \& Brennan, 2001) showed that following immunization with PE_PGRS 33 DNA vaccine, a significant humoral response was observed against recombinant PE_PGRS 33 and that the antibody response was directed against the Gly/Ala-rich PGRS domain. Moreover, this study showed that PE_PGRS 33 delivered as a DNA vaccine did not show protection in the mouse TB challenge model. In the present study, we extended our investigation to assess the potential of PE_PGRS 16 and PE_PGRS 26 DNA vaccines to show protection in the mouse TB challenge model, particularly since PE_PGRS 16 elicited different cellular responses, including the release of IL12 (see Figs 3 and 5), and contains an unusual C-terminal domain. Our results indicate that when given as DNA vaccines and studied in the mouse TB aerosol challenge model, none of the three PE_PGRS vaccines protect mice against aerosol challenge with the virulent $M$. tuberculosis Erdman strain as measured by colonization of the lungs and spleens of mice 30 days after challenge. In contrast, we have previously shown that a PE vaccine can protect in this TB model (Parra et al., 2006). Interestingly, for mice immunized with pe_pgrs 33 and to some extent pe_pgrs 26, disease was worsened as indicated by increased colonization of lungs in mice vaccinated with these PE_PGRS vaccines. In our in vitro studies, recombinant $M$. smegmatis expressing PE_PGRS 33 and PE_PGRS 26 survived better in macrophages that made less IL-12 and more IL-10 compared to 
M. smegmatis carrying the vector only. These data suggest that certain PE_PGRS antigens may induce Th2-like responses. This is supported by the observation that immunization with all three DNA vaccines induces antibodies that also show cross-reactivity with the PGRS domain of the three PE_PGRS antigens. However, expression of PE_PGRS 16 in our study induced release of IL-12, which is a key mediator of Th1 differentiation; this suggests that different PE_PGRS proteins may also elicit variable immune responses.

In the experiments described here, we demonstrate that the intracellular expression of different PE_PGRS proteins can alter the host cell environment, which can have consequences for both the mycobacteria and the host cells. Further studies are needed to determine why host cell responses to PE_PGRS 16 differ from those of PE_PGRS 33 and PE_PGRS 26. More generally, it will be of interest to determine how the expression of individual PE_PGRS genes is regulated in the host and what role this regulated control of PE_PGRS family members may have on the pathogenesis caused by M. tuberculosis.

\section{ACKNOWLEDGEMENTS}

We would like to thank Veerabadran Dheenadhayalan and Amy L. Yang for their useful discussions, Giovanni Delogu and Michael Niederweis for advice on methods for identifying cell surface proteins, and Sheldon Morris and Steven Derrick for critical reading of the manuscript. This work was supported by a grant from the National Vaccine Program Office of the US Department of Health and Human Services to M. J. B.

\section{REFERENCES}

Balaji, K. N., Goyal, G., Narayana, Y., Srinivas, M., Chaturvedi, R. \& Mohammad, S. (2007). Apoptosis triggered by Rv 1818c, a PE family gene from Mycobacterium tuberculosis, is regulated by mitochondrial intermediates in T cells. Microbes Infect 9, 271-281.

Banu, S., Honore, N., Saint-Joanis, B., Philpott, D., Prévost, M. C. \& Cole, S. T. (2002). Are the PE-PGRS proteins of Mycobacterium tuberculosis variable surface antigens? Mol Microbiol 44, 9-19.

Basu, S., Pathak, S. K., Banerjee, A., Pathak, S., Bhattacharyya, A., Yang, Z., Talarico, S., Kundu, M. \& Basu, J. (2007). Execution of macrophage apoptosis by PE_PGRS33 of Mycobacterium tuberculosis is mediated by Toll-like receptor 2-dependent release of tumor necrosis factor. $J$ Biol Chem 282, 1039-1050.

Brennan, M. J. \& Delogu, G. (2002). The PE multigene family: a 'molecular mantra' for mycobacteria. Trends Microbiol 10, 246-249.

Brennan, M. J., Delogu, G., Chen, Y., Bardarov, S., Kriakov, J., Alavi, M. \& Jacobs, W. R., Jr (2001). Evidence that mycobacterial PE_PGRS proteins are cell surface constituents that influence interactions with other cells. Infect Immun 69, 7326-7333.

Brennan, M. J., Espitia, C. \& Gey van Pittius, N. (2004). The PE and PPE multigene families of Mycobacterium tuberculosis. In Tuberculosis, 2nd edn, pp. 513-525. Edited by S. Cole, D. N. McMurray, K. Eisenach, B. Gicquel \& W. R. Jacobs. Washington, DC: American Society for Microbiology.

Cascioferro, A., Delogu, G., Colone, M., Sali, M., Stringaro, A., Arancia, G., Fadda, G., Palu, G. \& Mangenelli, R. (2007). PE is a functional domain responsible for protein translocation and localization on mycobacterial cell wall. Mol Microbiol 66, 1536-1547.

Chen, M., Gan, H. \& Remold, H. G. (2006). A mechanism of virulence: virulent Mycobacterium tuberculosis strain H37Rv but not attenuated $\mathrm{H} 37 \mathrm{Ra}$ causes significant mitochondrial inner membrane disruption in macrophages leading to necrosis. J Immunol 176, 3707-3716.

Cole, S. T., Brosch, R., Parkhill, J., Garnier, T., Churcher, C., Harris, D., Gordon, S. V., Eiglmeier, K., Gas, S. \& other authors (1998). Deciphering the biology of Mycobacterium tuberculosis from the complete genome sequence. Nature 393, 537-544.

Delogu, G. \& Brennan, M. J. (2001). Comparative immune response to PE and PE_PGRS antigens of Mycobacterium tuberculosis. Infect Immun 69, 5606-5611.

Delogu, G., Pusceddu, C., Bua, A., Fadda, G., Brennan, M. J. \& Zanetti, S. (2004). Rv1818c-encoded PE_PGRS protein of Mycobacterium tuberculosis is surface exposed and influences bacterial cell structure. Mol Microbiol 52, 725-733.

Delogu, G., Sanguinetti, M., Pusceddu, C., Bua, A., Brennan, M. J., Zanetti, S. \& Fadda, G. (2006). PE_PGRS proteins are differentially expressed by Mycobacterium tuberculosis in host tissues. Microbes Infect 8, 2061-2067.

Dheenadhayalan, V., Delogu, G. \& Brennan, M. J. (2006a). Expression of the PE_PGRS 33 protein in M. smegmatis triggers necrosis in macrophages and enhanced mycobacterial survival. Microbes Infect 8, 262-272.

Dheenadhayalan, V., Delogu, G., Sanguinetti, M., Fadda, G. \& Brennan, M. J. (2006b). Variable expression patterns of Mycobacterium tuberculosis PE_PGRS genes: evidence that PE_PGRS16 and PE_PGRS26 are inversely regulated in vivo. $J$ Bacteriol 188, 3721-3725.

Espitia, C., Laclette, J. P., Mondragón-Palomino, M., Amador, A., Campuzano, J., Martens, A., Singh, M., Cicero, R., Zhang, Y. \& Moreno, C. (1999). The PE-PGRS glycine-rich proteins of Mycobacterium tuberculosis: a new family of fibronectin-binding proteins? Microbiology 145, 3487-3495.

Flores, J. \& Espitia, C. (2003). Differential expression of PE and PE_PGRS genes in Mycobacterium tuberculosis strains. Gene 318, 75-81.

Heinz, C. \& Niederweis, M. (2000). Selective extraction and purification of a mycobacterial outer membrane protein. Anal Biochem 285, 113-120.

Jain, S. K., Satyaseela, M. P., Lamichhane, G., Kim, K. S. \& Bishai, W. R. (2006). Mycobacterium tuberculosis invasion and traversal across an in vivo human blood-brain barrier as a pathogenic mechanism for central nervous system tuberculosis. J Infect Dis 193, 1287-1295.

Parra, M., Cadieux, N., Pickett, T., Dheenadhayalan, V. \& Brennan, M. J. (2006). A PE protein expressed by Mycobacterium avium is an effective T-cell immunogen. Infect Immun 74, 786-789.

Pfaffl, M. W. (2001). A new mathematical model for relative quantification in real-time RT-PCR. Nucleic Acids Res 29, e45.

Ramakrishnan, L., Federspiel, N. A. \& Falkow, S. (2000). Granulomaspecific expression of Mycobacterium virulence proteins from the glycine-rich PE-PGRS family. Science 288, 1436-1439.

Talaat, A. M., Lyons, R., Howard, S. T. \& Johnston, S. A. (2004). The temporal expression profile of Mycobacterium tuberculosis infection in mice. Proc Natl Acad Sci U S A 101, 4602-4607.

Talarico, S., Cave, M. D., Marrs, C. F., Foxman, B., Zhang, L. \& Yang, Z. (2005). Variation of the Mycobacterium tuberculosis PE_PGRS33 gene among clinical isolates. J Clin Microbiol 43, 4954-4960.

Talarico, S., Zhang, L., Marrs, C. F., Foxman, B., Cave, M. D., Brennan, M. J. \& Yang, Z. (2008). Mycobacterium tuberculosis 
PE_PGRS 16 and PE_PGRS 26 genetic polymorphism among clinical isolates. Tuberculosis (Edinb) 88, 283-294.

Voskuil, M. I., Schnappinger, D., Rutherford, R., Liu, Y. \& Schoolnick,

G. K. (2004). Regulation of the Mycobacterium tuberculosis PE/PPE genes. Tuberculosis (Edinb) 84, 256-262.
Williams, E. P., Lee, J. H., Bishai, W. R., Colantuoni, C. \& Karakousis, P. C. (2007). Mycobacterium tuberculosis Sig F regulates genes encoding cell wall associated proteins and directly regulates the transcriptional regulatory gene phoY1. J Bacteriol 189, 4234-4242.

Edited by: M. Daffé 